\title{
Konrad Geßner als Botaniker
}

\author{
Von Heinrich Zoller, Basel
}

Noch im 16. Jahrhundert haben die meisten Gelehrten die Pflanzen nur als Träger von Heilkräften betrachtet, weshalb sich die Botanik nur langsam und verhältnismäßig spät als selbständige Wissenschaft von der Medizin loslöste. Trotzdem hat sich schon in der Zeit der Renaissance eine geistige Entwicklung angebahnt, welche die Grundlagen schuf für neuartige Forschungsmethoden in der Botanik. Neben der von LuGA GHINI ${ }^{1}$ entdeckten Möglichkeit, getrocknete Pflanzen in Herbarien aufzubewahren, führte seit Leonardo da Vinci und Albrecht Dürer das Streben nach naturgetreuen Abbildungen zu erhöhter objektiver Beobachtung und diese mehr und mehr zur ausschließlichen Beschäftigung mit den Pflanzen um ihrer selbst willen. Damit wuchs die Möglichkeit gegenseitigen Erfahrungsaustausches, von Sammelreisen in die Gebirge und in abgelegene Gegenden. In der Folge setzte sich die Erkenntnis durch, daß die Pflanzenwelt von Gebiet zu Gebiet wechselt und somit entsprechende Beschreibungen für manche einheimische Gewächse Mitteleuropas bei den griechischen Autoren, wie Dioscorides und Theophrast, gar nicht zu finden waren. In diese Periode fallen die ersten Versuche, Pflanzen nach einer wissenschaftlichen Methode zu beschreiben und sie nach allgemein anwendbaren Grundsätzen zu benennen. Der Gedanke, die Mannigfaltigkeit nach gestaltlicher Ähnlichkeit und Verwandtschaft zu ordnen, begann die Gelehrten zu beschäftigen. Ohne daß mit dieser Entwicklung die Botanik im 16. und 17. Jahrhundert schon zu einer auch äußerlich selbständigen Wissenschaft geworden wäre, hat sie doch durch diese neue Zielsetzung die rein scholastische Methode des bedingungslosen Zurückgehens auf die Antike schrittweise überwunden.

Seit seiner frühesten Kindheit, als er von Johannes Frick ${ }^{2}$ die ersten botanischen Anregungen erhielt, hat sich Konrad Geßner mit stets steigendem Eifer dem Studium der Pflanzen gewidmet, bis diese Tätigkeit in den letzten Jahren vor seinem Tode sein ganzes Leben ausgefüllt hat. Eine Würdigung der universellen Persönlichkeit Konrad Geßners ist deshalb

1 Luca Ghini, von 1534 bis 1544. Professor der Medizin und Botanik an der Universität Bologna.

2 Johannes Frick, Onkel der Mutter von Konrad Geßner, Kaplan am Großmünster in Zürich. 
ohne seine grenzenlose, keine Opfer scheuenden Hingabe an die Beobachtung der Pflanzen unmöglich. Auch wenn sein geplantes Werk, die Historia plantarum, in tragischer Weise unvollendet geblieben ist, so darf, ohne zu übertreiben, doch gesagt werden, daß die einmalige Forscherpersönlichkeit Konrad Geßners nirgends so ergreifend zu den Nachfahren spricht wie aus den hinterlassenen, kolorierten Zeichnungen zu seinem «Pflanzenbuch» ${ }^{3}$.

Es ist auffallend, wie verschieden später der Einfluß beurteilt worden ist, den Konrad Geßner auf die Entwicklung der Botanik zur selbständigen, biologischen Wissenschaft seit dem 16. Jahrhundert ausgeübt hat. Als groBer Quellenkritiker ist Albrecht von Haller (1772) nach etwa zweihundert Jahren der Ansicht, Konrad Geßner habe als erster das richtige Prinzip der botanischen Methode erkannt, Geschlechter aufzustellen, die eine Anzahl Spezies umfassen, und Klassen als Inbegriff mehrerer Geschlechter. Diese hohe Einschätzung der Verdienste Konrad Geßners um die wissenschaftliche Botanik findet sich auch bei Kurt Sprengel (1817) und bei Ernst H.F. Meyer (1856). Nach Julius Sachs (1875) ändert sich in der zweiten Hälfte des 19. Jahrhunderts das Urteil über Konrad Geßner. Seine Verdienste geraten mehr und mehr in Vergessenheit, und als erste Botaniker, welche die rein praktischen Gesichtspunkte der Pflanze als Heil- und Nutzmittel abgelegt und die «natürliche Verwandtschaft» in den Vordergrund gerückt haben, gelten nun Caesalpin, Lobelius und Bauhin. In neuerer Zeit ist Konrad Geßner von botanischer Seite kaum mehr beachtet worden. Als Nichtbotaniker kommt allerdings auch WiLly Ley (1929) zum Schluß, daß Konrad Geßner in der Pflanzenkunde bahnbrechend war und in der Botanik bei längerem Leben wahrscheinlich LinNés spätere Tat vorweggenommen hätte.

Auf der Universitätsbibliothek Erlangen sind zwei Codices erhalten geblieben, welche die wesentlichsten Vorarbeiten Konrad Geßners zur geplanten Historia plantarum enthalten, die den zweiten Teil seines gigantischen Versuches bilden sollte, die gesamte Naturwissenschaft in einem großangelegten Werke darzustellen ${ }^{3}$. Bernhard Milt (1936) hat nach

3 Für Konrad Geßners Nachlaß ist nach wie vor grundlegend: D. C.C.Schmiedel, Conradi Gesneri ... opera botanica ... I und II, Norimbergae 1751-1771. Vom ganzen Nachlaß Geßners ist in diesem mustergültigen Werk durch Schmiedel ungefähr ein Drittel herausgegeben worden. Die auf der Universitätsbibliothek Erlangen befindlichen zwei Codices mit Geßners Nachlaß enthalten auf etwa 490 Folioseiten die Originale zu ungefähr 1500 Pflanzenbildern. Den gesamten in Erlangen noch vorhandenen Nachlaß besitzt die Zentralbibliothek Zürich in Form von Photographien und Photokopien. 
langer Verschollenheit die darin enthaltenen Originalzeichnungen von Pflanzen sehr eingehend untersucht. Nach Bernhard Milt (1947) hat Konrad Geßner als Begründer der naturwissenschaftlichen Morphologie zu gelten, erfaßte Geßner als erster den Begriff von Genus und Spezies und wußte, daß zur Art eine erbmäßige Konstanz gehört ${ }^{4}$. Für Bernhard Milt (1947) besteht kein Zweifel, daß Konrad Geßner in seiner Bemühung um die Pflanzen weit über einen bloßen Kräutersammler hinausgewachsen ist, daß seine Fragestellung und sein Forschungsziel rein wissenschaftlich waren.

4 Vgl. hiezu z. B. die entsprechenden Äußerungen in: Gesneri epist. medic. fol. 94 a und 86 a.

Erklärung zu den Abbildungen

Abb. 1. Cirsium tuberosum (L.) All. ... Cardui genus in Albio monte (ubi a villa Kolbenhof ad sinistram deflectendo ascendit, in summo forte quaternie ascendi illic potui ... - Über diesem Text die Jahreszahl 1563. Im Sinne der bei Geßner beginnenden morphologischen Analyse ist die Darstellung einer einzelnen, aus dem Köpfchen herauspräparierten Blüte besonders bezeichnend.

Abb. 2. Ranunculus repens L. (unten). Ranunculus acer L. ssp. Steveni (Andrz.) Hartm. (Mitte und links). aff. Ranunculus nemorosus DC. (geflecktes Blatt). - Neben der künstlerisch und wissenschaftlich vollendeten Darstellung der vegetativen Organe überrascht im Vergleich zu den Zeichnungen von Geßners Korrespondenten die exakte Wiedergabe des apokarpen Gynöceums, das aus zahlreichen freien Fruchtblättern besteht.

Abb. 3. Cardamine heptaphylla (Vill.) O.E.Schulz. - Auch hier findet sich der Versuch, die Früchte und ihren Öffnungsmechanismus genau festzuhalten. Er darf im Falle der Cruciferen-Schote nicht als gelungen betrachtet werden. Darüber war sich auch Geßner bewußt. So befindet sich auf einem anderen Blatt seines Nachlasses eine bessere Darstellung dieser Verhältnisse, in der bereits die falsche Scheidewand zu sehen ist. Besondere Bewunderung verdient die Art, wie Geßner die unterirdischen Schuppenrhizome beobachtet hat, ohne von ihrer Sproßnatur etwas zu wissen. Selbst die Oberblattrudimente und ihre Wachstumskrümmungen sind richtig getroffen. 


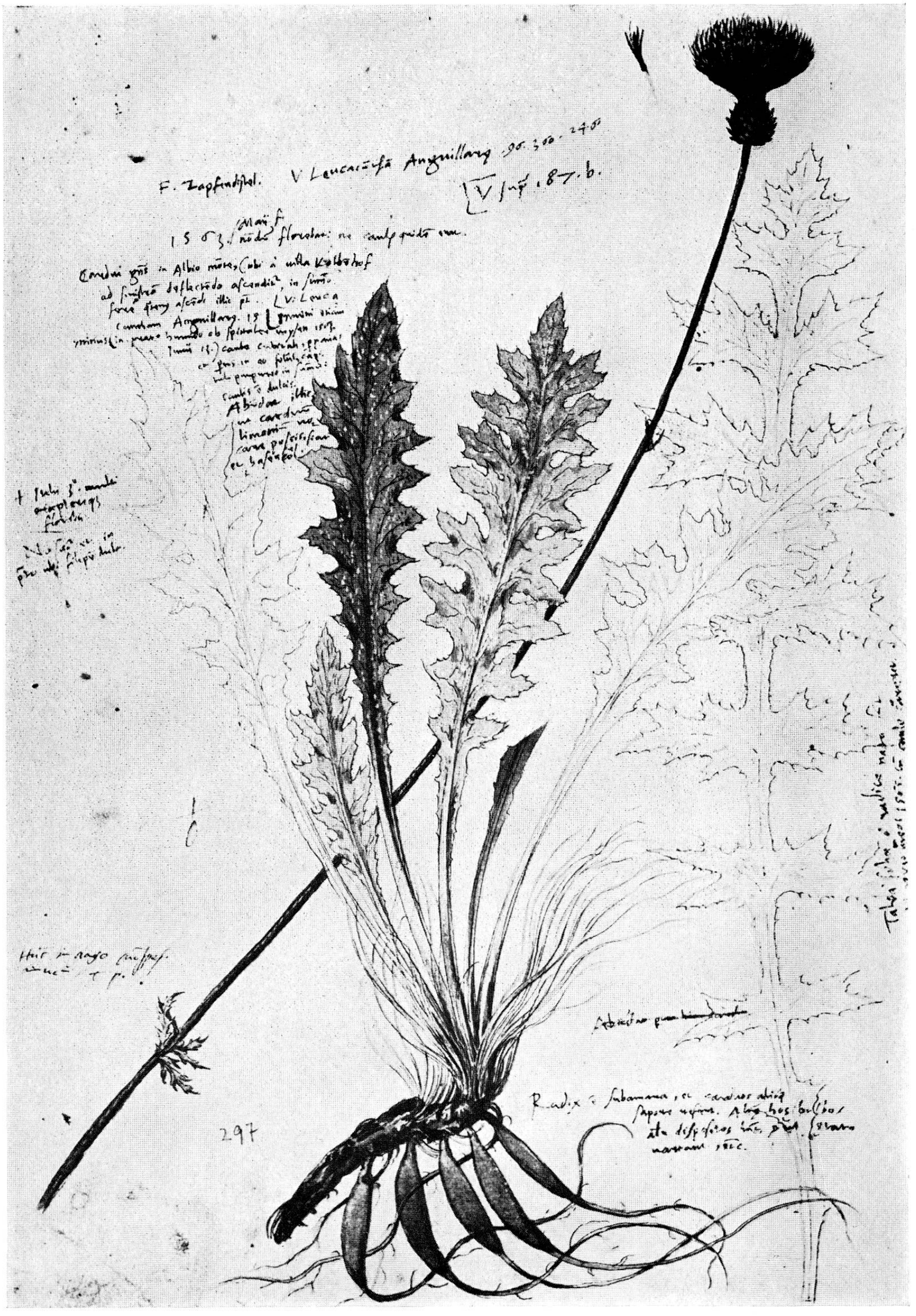




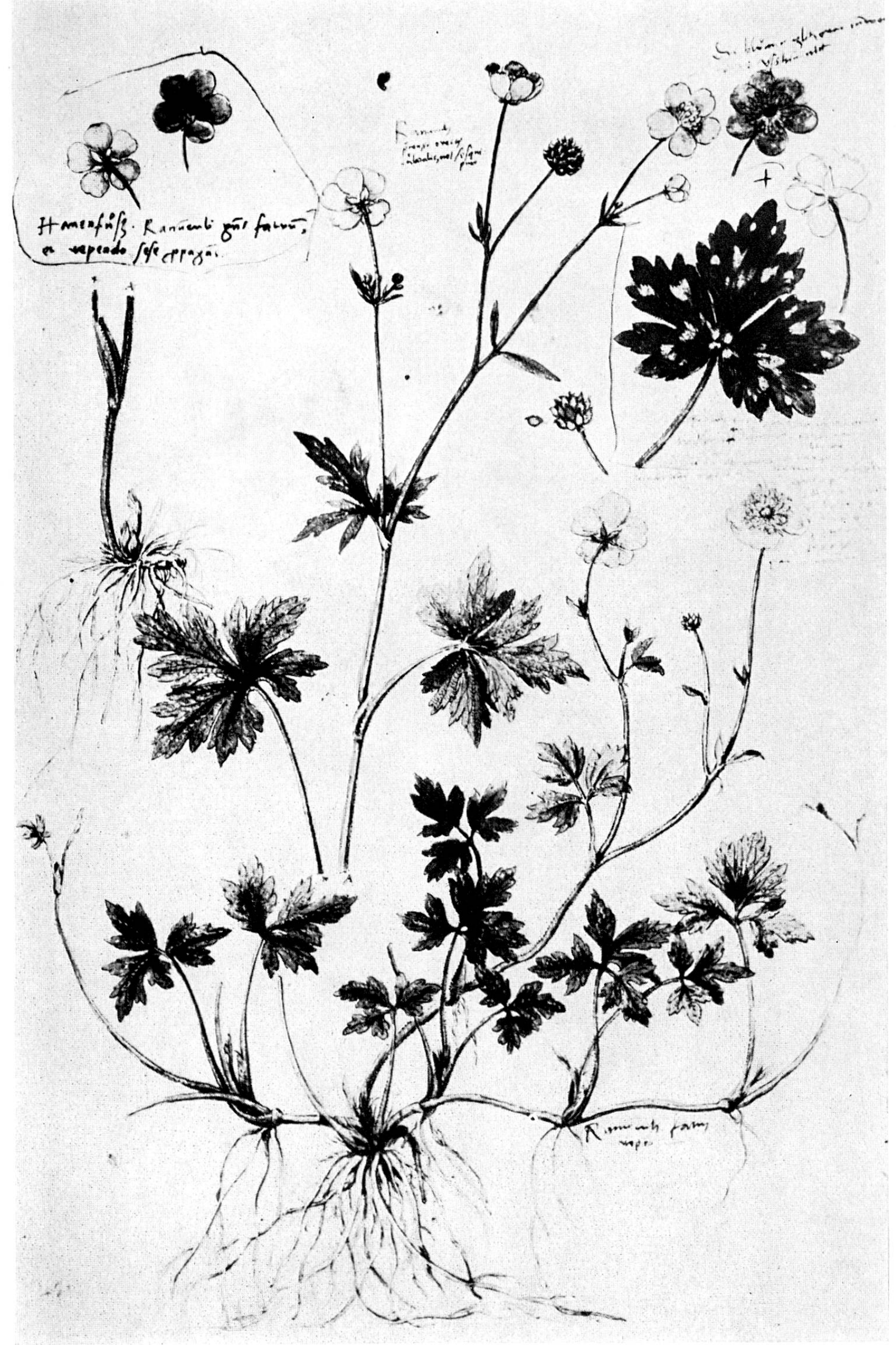




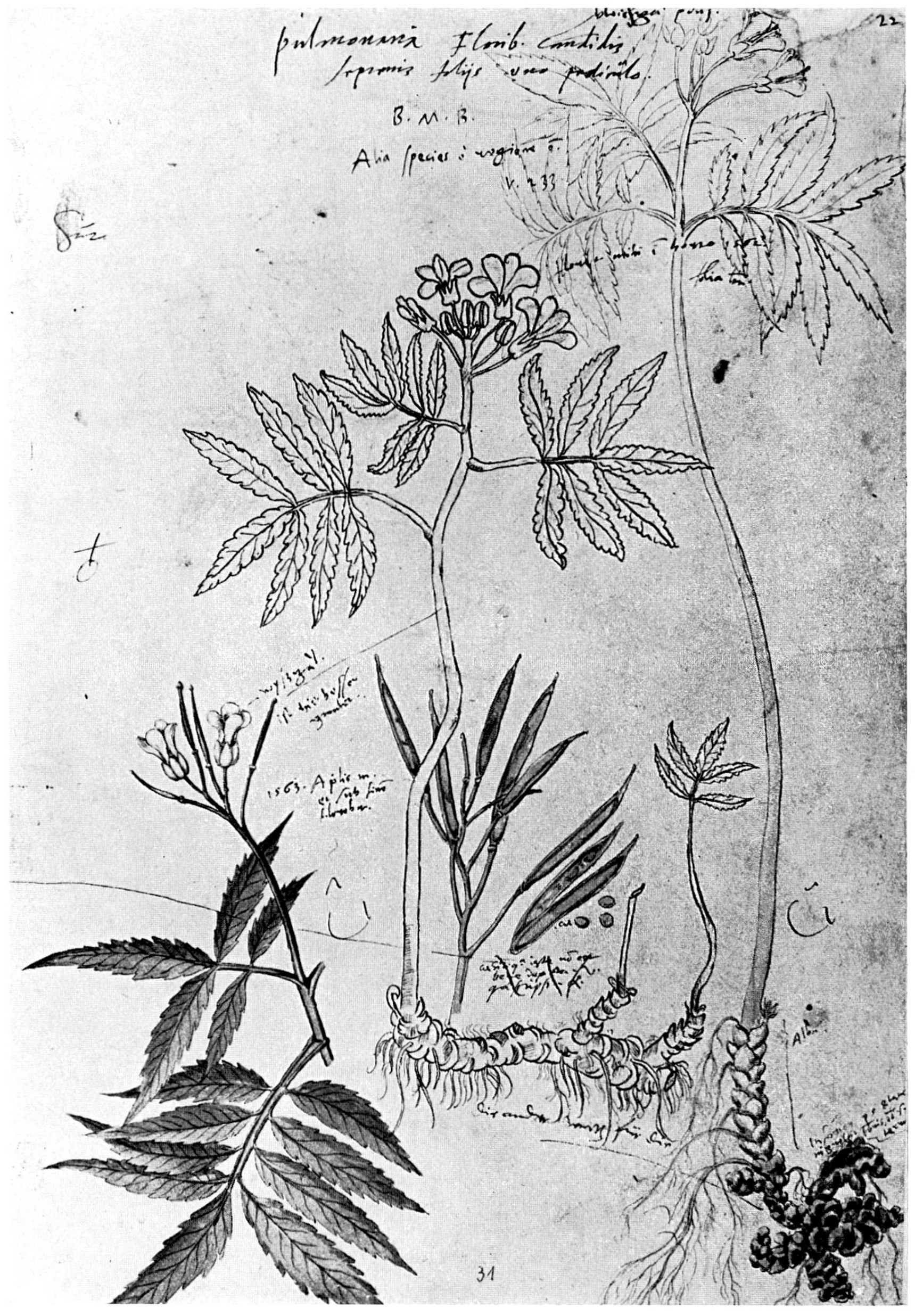


Es ist ein eigenartiges Zusammentreffen, daß im gleichen Jahre und in der gleichen Zeitschrift Beiträge zur Geschichte der botanischen Systematik erschienen sind, in deren erstem Teil, der die Periode von den Kräuterbüchern bis zu Carl von Linné behandelt, Konrad Geßner mit keinem Wort erwähnt und ausgerechnet das Verdienst, die Aufmerksamkeit auf die Merkmale der Früchte gelenkt zu haben, A. Caesalpino ${ }^{5}$ und die Blüte als Ganzes betrachtet zu haben, J. RAY ${ }^{6}$ zugeschrieben wird (WALTER RYTz und Charles Baenni, 1947). Nur ein Jahr später erscheint das schöne Buch Konrad Geßner als Gärtner von Diethelm Fretz (1948). Es mag mit der besonderen Problemstellung von Diethelm Fretz zusammenhängen, wenn er Konrad Geßner fast ausschließlich nur als Praktiker sieht. Nach Diethelm Fretz stelle Konrad Geßner seine gesamten Anstrengungen, das Walten der Natur zu ergründen und das überlieferte Wissen um dasselbe zu sammeln, bewußt oder unbewußt, immer in engste Beziehung zur ärztlichen Praxis und nutzbaren Heilmittellehre. Weiter steht bei Dieshelm Fretz zu lesen, daß Konrad Geßner nie Wissenschaft an und für sich, weder auf dem Gebiete der Zoologie und Botanik noch auf dem der Philologie und Bibliographie, getrieben habe. Diese Aussagen stehen in diametralem Widerspruch zu den Ansichten, die kurz zuvor von Bernhard MıLt geäußert worden sind. Es lohnt sich deshalb, anläßlich des 400. Todestages von Konrad Geßner, erneut zu untersuchen, welche Impulse die botanische Wissenschaft durch ihn empfangen hat.

Um die offensichtlichen Unstimmigkeiten der verschiedenen Meinungen hierüber zu klären, scheint es notwendig, strenger als bisher zwischen der verschiedenen Wirkung zu unterscheiden, die Konrad Geßners Briefwechsel und die zu seinen Lebzeiten erschienenen Publikationen einerseits und sein unveröffentlichter oder erst viel später gedruckter Nachlaß andererseits auf die botanische Fachwelt in der zweiten Hälfte des 16. Jahrhunderts ausgeübt haben. Nur die vor seinem Tode erschienenen Schriften und versandten Briefe konnten einen unmittelbaren Einfluß auf die zeitgenössischen Botaniker haben, während die Gelehrten erst zweihundert Jahre später einen Teil jener einzigartigen Pflanzenbilder zu Gesicht bekamen, von denen Konrad Geßner vor seinem Ableben schon über 1500 beisammen hatte ${ }^{3}$.

5 Andrea Caesalpino (1519-1603) von Arezzo, Schüler von Luca Ghini, Professor in Pisa und Leibarzt von Papst Clemens VIII.

6 John RaY (1628-1705) von Black Notlay in Essex. 
Sämtliche botanischen Publikationen, die Konrad Geßner vor seinem Tode veröffentlicht hat, besitzen eine eminent praktische Bedeutung. Schon seine erste botanische Schrift, Enchiridion historiae plantarum (1541), ein Kompendium antiker Pflanzenbeschreibungen aus Dioscorides, Paulus Aegineta, Theophrast, Plinius u. a. mit Angaben über die medizinischen Eigenschaften ist aus solchen Bedürfnissen heraus entstanden. Fortan sollte es nicht mehr nötig sein, kostbare Quellenschriften oder schwere Bündel mit Auszügen auf Exkursionen mitzuschleppen oder sich aus Bequemlichkeit auf die Zuverlässigkeit des Gedächtnisses zu stützen. Nachdem das gleiche Werk in stark verkleinertem Format bei Melchior Sessa in Venedig erschienen war, verfügten sämtliche Fachkollegen über ein erstes handliches botanisches Taschenbrevier.

Als großangelegter Versuch zu einer dringend notwendig gewordenen Konkordanz über die zahllosen, verwirrenden botanischen Namen, Begriffe und Ausdrücke wurde der Catalogus Plantarum (1542) konzipiert, wobei neben Griechisch und Latein auch Deutsch und Französisch sowie die Berufssprache der Apotheker berücksichtigt waren. Auch bei der Herausgabe des Lexicon rei herbariae trilingue (1553) zeigte sich Konrad Geßners Sinn für das Praktische, indem er diesem lateinisch-griechisch-deutschen Verzeichnis von Pflanzennamen, das David Kyber ${ }^{7}$ in alphabetischer Reihenfolge angelegt hatte, gleich ein weiteres hinzufügte, in dem er die verschiedenen aufgeführten Heilkräuter entsprechend der Sammelreife von Blüte, Frucht und Wurzel usw. in besonderen zwölf Monatslisten gruppierte. Konrad Geßner hat damit KyBers Werk zu einem für den täglichen Gebrauch geeigneten Handwörterbuch umgestaltet, dessen Erscheinen bewirkte, daß zahlreiche Ärzte und Apotheker seinen Kontakt suchten und mit ihm in Briefwechsel und Pflanzenaustausch traten.

Konrad Geßners Bestrebungen, die Kommunikation zwischen den botanisch interessierten Gelehrten zu erleichtern, erreichten den Höhepunkt mit der Herausgabe seines letzten größeren botanischen Werkes, Horti Germaniae (1561), in dem Diethelm Fretz (1948) zu Recht den ältesten Pflanzen- und Samenaustauschkatalog erblickt und das gleichzeitig für die einzelnen Arten praktische Hinweise über Aussaat und Kultur enthält.

Offensichtlich sind die von Konrad Geßner selber publizierten, wichtigeren botanischen Werke in engem Zusammenhang mit der medizinischen Heilmittellehre geschrieben worden und enthalten nur wenig von einer

7 David Kyber (1525-1553) von Straßburg, Freund von Konrad Geßner. 
selbständigen, rein wissenschaftlichen Problemstellung. Sie entstanden letztlich aus dem ständigen, an Konrad Geßner so sympathischen Bedürfnis, anderen Gelehrten zu helfen, die wissenschaftliche Arbeit allgemein zu erleichtern und dadurch den Fortschritt zu beschleunigen. Wenn die damaligen Fachkollegen diese Publikationen besonders hoch eingeschätzt haben, so deshalb, weil darin der Zweck verfolgt wurde, die notwendigen Grundlagen für eine bessere Verständigung zu schaffen. In dieser Hinsicht gleicht Konrad Geßners Streben den Absichten von CARL von LinnÉ8.

Es wäre jedoch verfehlt, aus dem Charakter dieser Schriften zu schließen, bei Konrad Geßners botanischen Untersuchungen seien die nützlichen Erwägungen der medizinischen Praxis stets im Vordergrund gestanden. Schon sein berühmter Brief an Avienus in Glarus ${ }^{9}$, mit dem er seinen Libellus de lacte (1541) eingeleitet hat, spricht deutlich gegen die Behauptung von Diethelm Fretz (1948), für Konrad Geßner sei die Betätigung als Gärtner nie Ausdruck des Wunsches gewesen, eine Welt der Augenweide, ein Gefilde erquickender Sinnenlust um sich zu haben. Im Gegenteil, Konrad Geßners Begeisterungsfähigkeit für die Natur an sich war so außergewöhnlich, daß er geradezu eine Betrachtung der Naturschönheiten für das Wohlergehen der Menschenseele für notwendig gehalten hat. Seine unverdorbene Erlebnisfreudigkeit ist auch auf jeder Seite der Descriptio montis Fracti (1555) zu spüren. Schon im 16.Jahrhundert hat Konrad Geßner wohl als erster das Hochgebirge als rein, schön, erhaben und erquickend empfunden und seiner Ergriffenheit beschwingten sprachlichen Ausdruck verliehen. Ebenfalls als erster ist er zweihundert Jahre vor Albrecht von HaLler auf die Höhenstufen der Vegetation im Gebirge aufmerksam ge-

8 Bernhard Milt (1947) geht aber entschieden zu weit, wenn er schreibt: «Schon lange vor LiNNÉ pflegte er (Konrad Geßner) eine binäre Nomenklatur mit Bezeichnung von Genus und Spezies; die ganze Sätze beanspruchenden Diagnosen wurden erst später in die Botanik eingeführt.» Eigenhändig geschriebene Polynome sah ich öfters auf Geßners Originalzeichnungen. Von einer konsequent durchgeführten Nomenklatur im Sinne von LiNNÉ kann trotz mancher binärer Namen jedenfalls noch keine Rede sein.

9 «...Diese erstaunenswürdige Höhe macht auf die Seele den Eindruck der Erhabenheit und reißt sie zur anbetenden Bewunderung des allweisen Schöpfers hin. Nur Menschen von träger Seele bewundern nichts, bleiben in dumpfer Gefühllosigkeit zu Hause, treten nicht heraus in den herrlichen Schauplatz der Welt, liegen gleich Murmeltieren schlummernd in einem Winkel begraben, bedenken es nicht, daß das menschliche Geschlecht auf diese Erde hingesetzt ist, um aus der Betrachtung ihrer Wunder etwas Größeres, nämlich die unsichtbare Gottheit selbst, kennenzulernen ...» 
worden, die er in ebenso origineller wie zutreffender Weise auf die mit zunehmender Meereshöhe sich verkürzende Vegetationszeit zurückführt ${ }^{10}$.

Zahlreiche Stellen seines Briefwechsels beweisen, daß Konrad Geßner viel Zeit und Wert auf eine wirklichkeitstreue Darstellung sämtlicher Pflanzenorgane, insbesondere der Blüten, Früchte und unterirdischen Teile legte, ja sogar kein Opfer scheute, dieses Ziel zu erreichen (vgl. z.B. Ernst H.F.Meyer, 1856, Band III, S. 333). Selbst jene zwölf Tafeln in SchmieDELS posthumer Ausgabe ${ }^{3}$, die insgesamt 198 kolorierte Kupferstiche enthalten, vermitteln noch deutlich alle Vorzüge Geßnerscher Meisterschaft, auch wenn sie keinen vollständigen Eindruck von der wissenschaftlichen und künstlerischen Qualität mancher Originalzeichnungen vermitteln. Sicher steht fest, daß Konrad Geßner von zahlreichen Gewächsen die ersten exakten morphologischen Analysen ihrer Blütenblätter, Staubgefäße, Früchte und Samen, ja sogar von Ausläufern, Rhizomen und anderen Speicherorganen geliefert hat. Das grundsätzlich Neue und der bedeutende Fortschritt in Geßners Pflanzenabbildungen beruht auf seinem Prinzip, neben einem allgemeinen Habitusbild die morphologisch wichtigsten Organe, aus ihrem Verbande losgelöst, einzeln und stark vergrößert wiederzugeben $^{11}$. In dieser Hinsicht lassen sie die kaum dreißig Jahre älteren, künstlerisch ebenbürtigen Pflanzenaquarelle des Hans Weiditz ${ }^{12}$, die Vor-

10 «... So können wir also die Hochgebirge der Alpen in vier Regionen einteilen. Auf der obersten Höhe herrscht ein beständiger Winter, Schnee, Eis und kalte Winde. Dann folgt die Frühlingsgegend, nach einem sehr langen Winter ein sehr kurzer Frühling. Dann die herbstliche Lage, in welcher drei Jahreszeiten vorkommen, Winter, Frühling und etwas vom Herbste, und endlich die unterste Tiefe, wo auch ein kurzer Sommer sich findet, also alle vier Jahreszeiten ...»

11 Die Qualität der Originale ist freilich sehr verschieden. Beim Durchgehen der Photokopien in der Zentralbibliothek in Zürich stellte sich jedoch heraus, daß die mittelmäßigen und schlechten keine morphologischen Einzeldarstellungen neben dem Habitusbild aufweisen, die guten bis vorzüglichen dagegen mit solchen Detailanalysen versehen sind. Es dürfte deshalb möglich sein, an Hand dieser Unterschiede herauszubekommen, welche Abbildungen von Geßner selbst stammen oder doch unter seiner Anleitung entstanden sind und welche ihm von Korrespondenten, wie Dalechamp, Kentmann, Occo und anderen zugesandt wurden. Herrn Dr. Rudolf Steiger sei an dieser Stelle für seine Ratschläge bei der Durchsicht bestens gedankt.

12 Hans Weiditz, von Straßburg. Seine Pflanzenaquarelle aus dem Jahre 1529 sind die Originalvorlagen zu den Holzschnitten im Kräuterbuche von OtTo BRUNFELs. WALTER RYtz (1936) hat darauf hingewiesen, daß schon Hans Weiditz öfters neben einem Habitusbild einzelne Teile der betreffenden Pflanzen wiederholt hat, und es ist durchaus möglich, daß Konrad Geßner bei Felix Platter die Weiditzschen Pflanzenaquarelle 
lagen zum Kräuterbuch von Отto BRunfels ${ }^{13}$ weit hinter sich. Vergleichbar sind sie dagegen mit Leonardo da Vincis anatomischen Aufzeichnungen, und in manchen Fällen, wie etwa von den Wurzelstöcken der Zahnwurzarten, gibt es keine gleichwertigen oder besseren Darstellungen mehr bis ins 19. Jahrhundert oder sogar bis zu Wilhelm Troll.

Ist deshalb Konrad Geßner mit Bernhard Milt (1947) als Begründer der naturwissenschaftlichen Morphologie anzusehen? Zweifelsohne hat Konrad Geßner in seinen Zeichnungen als erster die erste Stufe wirklicher morphologischer Untersuchungsmethodik erreicht und vor allem in seinen letzten Lebensjahren bewußt und konsequent angewendet. Er war damit seinen Zeitgenossen um mindestens ein bis zwei Jahrhunderte voraus. Einen entscheidenden Impuls haben aber Geßners morphologische Beobachtungen auf die weitere Entwicklung der Botanik im ausgehenden 16. Jahrhundert nicht genommen. Auch wenn Konrad Geßner den hervorragenden Wert der Blüten und Früchte für die Bestimmung der Verwandtschaft zu seiner Zeit wie kein zweiter erkannt und noch zu seinen Lebzeiten in brieflichen Äußerungen oft bekräftigt hat, so vermochte er nicht zu verhindern, daß noch bis weit ins 17. Jahrhundert hinein die verwandtschaftliche Gruppierung im Pflanzenreich mehr nach dem Habitus oder nach einem undefinierbaren Instinkt vollzogen wurde als nach den Ursachen der wahrgenommenen Ähnlichkeit.

Als in der Mitte des 18. Jahrhunderts Schmiedel endlich die Geßnerschen Abbildungen nach einer zweihundert Jahre langen Wartezeit veröffentlichte, wurde die verspätete Publikation wohl bewundert und bestaunt, blieb jedoch ohne jeden Nutzen für die von der neuen Klassifikation durch Carl von Linné beherrschten biologischen Wissenschaft, die damit beschäftigt war, mit den Traditionen früherer Jahrhunderte zu brechen.

Schwer verständlich bleibt die Tatsache, wie wenig von Konrad Geßners Meisterschaft auf seine berühmten Basler Freunde Felix Platter ${ }^{14}$ und

gesehen hat und von dort dieses Verfahren der Einzeldarstellung übernahm. Was jedoch bei Weiditz mehr zur künstlerischen Ausgestaltung gehört, das erscheint bei Konrad Geßner plötzlich als wissenschaftlich-morphologische Analyse, so das Erkennen des Oberblattrudimentes an den zuletzt gebildeten Schuppen am Zahnwurzrhizom, jene herrliche, genaue Untersuchung über die verkümmerten Staubgefäße der Gratiola, die Darstellung von Spaltfrüchten bei Umbelliferen und von einzelnen Blüten des Kompositenköpfchens, usw.

13 Oтto Brunfels (1500-1534) von Mainz, Lehrer und Arzt in Straßburg.

14 Felix Platter (1536-1614), Professor der Medizin in Basel. Er besaß ein berühmtes Naturalienkabinett, aus dem uns eines der ältesten Herbarien erhalten geblieben ist, 
Johannes Bauhin ${ }^{15}$ übergegangen ist. Selbst nachdem auch Andrea Caesalpino in De plantis libri XVI (1583) ebenfalls auf die Bedeutung der Früchte und Samen für die Systematik hingewiesen hat, bleiben bei CASPAR BAUHIN ${ }^{16}$ diese noch weitgehend vernachlässigt. Während aber nach Caesalpino eine Klassifikation a priori aus philosophischen Erwägungen auf Grund der Früchte und Samen stattfinden mußte, so gelangte Geßner durch konkrete morphologische Beobachtung und naturgetreue Wiedergabe seiner Analyse schon vor Caesalpin zur gleichen Erkenntnis. CaEsaLPINos System war damals durchaus zeitgemäß und hat als großangelegte Publikation bis ins 18. Jahrhundert nachgewirkt, Geßners unvollendetes Werk war im besten Sinne modern, blieb aber seinen Zeitgenossen vorenthalten.

in dem sich auch eine Reihe Weiditzscher Aquarelle befindet (vgl. Walter Rytz, 1933 und 1936).

15 Johannes Bauhin (1541-1613), älterer Bruder von Caspar Bauhin.

16 Caspar Bauhin (1560-1624), Professor der Anatomie und Botanik in Basel.

\section{Literatur}

Fretz Diethelm, Kontad Geßner als Gärtner, Zürich 1948.

Haller Albrecht von, Bibliotheca botanica, Nürnberg 1772.

Hanhart Johannes, Conrad Geßner, Winterthur 1824.

LEY Willy, Konrad Gesner, München 1929.

Meyer Ernst H.F., Geschichte der Botanik, Königsberg 1856.

Milt Bernhard, Konrad Geßners Historia Plantarum (Fragmenta relicta), Vjschr. Naturf. Ges. Zürich 81 (1936).

- - 1947, Zürichs Vergangenheit in Naturwissenschaft und Medizin (Mittelalter bis 16. Jahrhundert), Gesnerus 4 (194.7) Fasc. 1.

Rytz Walter, Das Herbarium Felix Platters, Verh. Naturf. Ges. Basel 44 (1933) 1. Teil. - - Die Pflanzenaquarelle des Hans Weiditz aus dem Jahre 1529, Bern 1936.

- - und Charles Baehni, Beiträge zur Geschichte der botanischen Systematik, Gesnerus 4 (1947) Fasc. 3/4.

SACHS Julius, Geschichte der Botanik, München 1875.

SPrengel Kurt, Geschichte der Botanik, Leipzig 1817. 